\title{
Design study of a "scintronic" crystal targeting tens of picoseconds time resolution for gamma ray imaging: the ClearMind detector
}

\author{
D. Yvon ${ }^{a}$, V. Sharyy ${ }^{a}$, M. Follin ${ }^{a}$, J-P Bard ${ }^{a}$, D. Breton ${ }^{b}$, J. Maalmi $^{b}$, C. Morel ${ }^{c}$, E. \\ Delagnes $^{a}$ \\ ${ }^{a}$ Univ Paris-Saclay, CEA, Institut de recherche sur les lois Fondamentales de l'Univers, 91191, Gif- \\ sur-Yvette, France \\ ${ }^{b}$ Univ Paris-Saclay, CNRS, Laboratoire Irène Joliot Curie, 91898, Orsay, France \\ ${ }^{c}$ Aix-Marseille Univ, CNRS/IN2P3, CPPM, Marseille, France \\ E-mail: dominique. yvonecea.fr
}

\section{ABSTRACT:}

We describe the concept of a new gamma ray "scintronic" detector targeting a time resolution of the order of 25 ps FWHM, with millimetric volume reconstruction and high detection efficiency. Its design consists of a monolithic large $\mathrm{PbWO}_{4}$ scintillating crystal with an efficient photocathode directly deposited on it. With an index of refraction higher for the photocathode than for the crystal, this design negates the total reflection effect of optical photons at the crystal/photo-detector optical interface, and thus largely improves optical coupling between the crystal and the photodetector. This allows to detect efficiently the Cherenkov light produced by $511 \mathrm{keV}$ photoelectric conversions in $\mathrm{PbWO}_{4}$, and to optimize the detector time resolution. Furthermore, the low-yield, fast scintillation light produced additionally by $\mathrm{PbWO}_{4}$ increases the detected photon statistics by a factor 10, thus fostering accurate ( 3 dimensional) localization of the gamma ray interaction within the crystal and providing a fair measurement of the deposited energy.

This paper lists the technological challenges that have to be overcome in order to build this "scintronic" detector. We show that all the key technologies have now been demonstrated and present results of a preliminary Monte Carlo simulation, which include an innovative event reconstruction algorithm to support the claimed performances of the detector.

KEYWORDS: Fast gamma ray detection; High-resolution gamma ray detector; Cherenkov detector; monolithic block detector; PET imaging; TOF-PET. 
I. Context 3

II. Detector development for PET 3

i. R\&D on spatial resolution 3

ii. R\&D on time resolution 4

III. ClearMind Detector Design 5

IV. Preliminary results Erreur! Signet non défini.

i. Photocathode on scintillating crystal 6

ii. $\quad$ Producing large size crystals 6

iii. Signal readout $\quad 6$

iv. Photocathode deposition on $\mathrm{PbWO}_{4}$ crystal 6

v. $\mathrm{PbWO}_{4}$ crystals light yield

$\begin{array}{lll}\text { vi. } & \text { MCP-PMT and transmission lines readout } & 7\end{array}$

vii. Fast readout amplifiers 8

\begin{tabular}{ll} 
viii. & SAMPIC waveform recorder \\
\hline
\end{tabular}

$\begin{array}{ll}\text { ix. Photodetector performance } & 9\end{array}$

V. Simplified detector Monte Carlo simulation 9

i. Preliminary event reconstruction 11

ii. Computed performance 12

$\begin{array}{ll}\text { VI. Discussion } & 14\end{array}$

Acknowledgments $\quad 14$

$\begin{array}{ll}\text { References } & 14\end{array}$ 


\section{Context}

Recently, the development of new types of ultra-fast compact photo-detectors has improved coincidence time resolution (CTR) of scintillation spectrometric chains below 100 ps FWHM. These detectors consist of fast, thin scintillators, typically $\mathrm{LSO}, \mathrm{LYSO}, \mathrm{LaBr}_{3}$ or $\mathrm{CeBr}_{3}$, optically coupled to SiPM matrices [1], [2], [3], [4], [5].

That is why a new, very ambitious CTR technological frontier appears at the edge of $10 \mathrm{ps} \mathrm{FWHM}$ [6], which would make it possible to foresee new ultra-fast gamma ray imaging applications. For example, in positron emission tomography (PET), an image could be acquired virtually without tomographic inversion with a 10 ps CTR time-of-flight (TOF)-PET camera.

Detector efficiency is limited by the stopping power of the scintillating material, which depends on its density, its thickness and its effective atomic number. Today, energy and spatial resolutions are limited by scintillation light yield and efficiency of light collection. Spatial resolution degrades additionally because of scattering and multiple reflections of optical photons in the crystal. Time resolution is limited by the shape of the scintillation light pulse, namely its rise and decay times, and the efficiency in collecting these scintillation photons. As such, the generation of a few dozen Cherenkov photons by photoelectric or Compton electrons is almost instantaneous compared to the production of scintillation light. The collection of both the Cherenkov photons and the scintillation photons is impacted by reflections on the crystal surfaces. In addition, time resolution of the detector is also limited by the uncertainty on depth-of-interaction (DOI) in thick crystals.

\section{Detector development for PET}

Detector developments for PET are numerous and rich. For example, the reader can refer to [7] for a general overview. Recent efforts have often been motivated by the following goals:

- combining PET and magnetic resonance imaging (MRI) together,

- improving spatial resolution of PET (often in the context of neurological or of small animal imaging),

- improving time resolution of TOF-PET camera that permits to reduce patient dose or scan duration, thus opening new opportunities in the field of low count imaging as for theranostics [8].

The technologies under development must achieve a delicate compromise between all the properties described in the previous paragraph, especially the efficiency constraint, sometimes aiming at several of these goals.

Most of these developments use inorganic scintillators with high photon yields coupled to semiconductor photo-multipliers (SiPMs). SiPMs provide excellent time resolution, have a high quantum efficiency of photodetection and retain good properties when placed in an intense magnetic field: a major requirement for PET-MRI combined scanners. They are relatively simple to use. Their main drawback is their large dark count rate, which implies either to use scintillators with high light yields, or to cool them down a lot. SiPMs have become the reference photodetector for many manufacturers and the replacement of PMTs by SiPMs allowed to improve TOF-PET scanner CTRs from $\sim 550$ ps FWHM [9], [10] down to $\sim 350$ ps FWHM [11], [12]. Very recently, the Biograph Vision scanner from Siemens achieved a CTR of 214 ps FWHM [13].

\section{i. $\quad R \& D$ on spatial resolution}

R\&D on spatial resolution represents a more limited effort. In nonTOF 3D PET, improving spatial resolution at constant image signal-to-noise ratio (SNR) requires to increase scan statistics by the fourth power of the spatial resolution improvement factor, e.g. by increasing geometrical acceptance of the scanner, which combines detection efficiency and detector angular coverage, or by increasing scan duration. As an example, the EXPLORER project proposed to extend the axial length of the scanner to $2 \mathrm{~m}$ to improve sensitivity [14]. It is a challenge that scintillator technologies are struggling to reach. Some breakthrough technologies using liquid detectors are proposed by the community of physicists. Pre-localization of the annihilation position by using TOF information [15] or three-photon imaging techniques [16] allows for reducing this constraint on scan statistics by a factor proportional to the ratio of the imaged object size to the pre-localization accuracy [17], [18]. 


\section{ii. $\quad R \& D$ on time resolution}

$\mathrm{R} \& \mathrm{D}$ on time resolution is now the main direction in research laboratories working on gamma ray detectors for PET. [19], and [6] present a complete review of the developments of detectors for TOFPET. If resolutions of $100 \mathrm{ps} \mathrm{FWHM} \mathrm{are} \mathrm{likely} \mathrm{to} \mathrm{be} \mathrm{demonstrated} \mathrm{within} \mathrm{the} \mathrm{coming} \mathrm{years,} \mathrm{improving}$ CTR by an order of magnitude would need to introduce a paradigm shift in PET instrumentation [20]. The sensors currently used for PET are far from achieving such a performance. This is due in particular to "non-optimal" light production yield, "too slow" scintillation rise and fall time constants, and to the significant thickness of crystals required for efficient detection (typically $2 \mathrm{~cm}$ ) of $511 \mathrm{keV}$ annihilation photons, which induces a dispersion in the light collection times. To overcome these limitations, the community proposed to:

- improve scintillator light yield: a long R\&D process,

- use Cherenkov light: a small number of photons but almost instantaneous,

- measure the depth-of-interaction (DOI) of the gamma ray within the crystal and decorrelate detection time from DOI.

The prototypes proposed by the laboratories use one or more of these options. For example, [21] and [22] have studied the possibility to use the Cherenkov light to improve time resolution of a "slow" BGO detectors. [23] has documented efficient $511 \mathrm{keV}$ gamma ray detection using Cherenkov light in a liquid detector. [24] have studied the performance of a $\mathrm{PbWO}_{4}$ cubic Cherenkov detector decorrelating DOI by Monte Carlo simulation, [25] published excellent time resolutions using MCP-PMT with leadglass optical windows, unfortunately with low $511-\mathrm{keV}$ detection efficiency.

[26] and $[27,28]$ have particularly studied the feasibility and relevance of time-of-flight detectors based on lead fluoride $\left(\mathrm{PbF}_{2}\right)$ crystals. A Cherenkov radiator was assembled with a micro-channel plate photo-multiplier tube (MCP-PMT) using an optical gel. [27] have grouped signals in 16 channels amplified by low noise amplifiers (mini-circuit, $40 \mathrm{~dB}$ ) and have read out these signals with the 32channels SAMPIC module. They measured a detection efficiency of $25 \%$ for a crystal $53 \times 53 \times 10 \mathrm{~mm}^{3}$ and a time resolution of $150 \mathrm{ps} \mathrm{FWHM}$ in the center of the detector. To pursue this progress, it became essential to calibrate the response of the photomultiplier and its complete readout chain over the entire detection surface [28]. This was achieved by using a pulsed laser with a light beam duration shorter than the MCP-PMT transit-time spread.

The potential of a total-body PET camera using $\mathrm{PbF}_{2}$ crystals was modeled with the GATE Monte Carlo simulation platform [29], [30], [31]. Results show that despite its modest efficiency, such a detector would offer an equivalent imaging quality, or even slightly better as compared to other existing cameras [32].

To get a significant improvement on time resolution, it is necessary either to use photomultipliers with better time resolutions (nothing exists better than MCP-PMT), or to improve significantly the collection efficiency of the Cherenkov light produced in $\mathrm{PbF}_{2}$. This second idea led the development of the ClearMind detector. Actually, the best way to improve optical coupling between the crystal and the photodetector is to deposit a photocathode directly on the crystal. Nevertheless, $\mathrm{PbF}_{2}$ is fragile and, in addition, degases lead compounds and fluorinated lead when heated to the temperatures necessary for the photocathode evaporation, which is not suitable.

Lead tungstate $\left(\mathrm{PbWO}_{4}\right)$ is a scintillating crystal that has been studied in detail for particle physics experiments [33], [34], [35], [36]. It has much better mechanical properties and degases considerably less than $\mathrm{PbF}_{2}$ at high temperature. For these reasons, it is considered to be a good candidate to have a bialkaly photocathode deposited directly on its surface. Its Cherenkov light production is close to $\mathrm{PbF}_{2}$ and it also generates a few scintillation photons $(\sim 300$ photons/MeV) at short time scales (a few nanoseconds). This increased light output, together with the improved optical coupling, will make it possible to measure the energy deposited in the crystal and to reconstruct the position of the gamma ray interactions, including in particular DOI.

We have also studied the possibility to use SiPMs as photo-detectors for $\mathrm{PbWO}_{4}$. As expected, the dark count rate of the SiPMs makes this task very difficult. The use of a SiPM to detect Cherenkov light requires either an external trigger, for example a coincidence trigger with a MCP-PMT signal, or the means to reduce dark count rate. 
Table 1: Main properties and targeted performances of a ClearMind detector.

\begin{tabular}{|c|c|}
\hline Specifications & Target performance @ 511 keV \\
\hline Spatial resolution & Down to few $\mathrm{mm}^{3}$ \\
\hline Time resolution & $25 \mathrm{ps}$ (FWHM) \\
\hline Detector efficiency & $\begin{array}{c}\text { Highest possible: } \mathrm{PbWO}_{4} \text { has a } 9 \mathrm{~mm} \text { attenuation length with } \\
\text { a photoelectric fraction of } 43 \% \text { at } 511 \mathrm{keV}\end{array}$ \\
\hline \multicolumn{2}{|c|}{ Plug and play once developed } \\
\hline
\end{tabular}

\section{ClearMind Detector Design}

To meet the specifications given in Table 1, we propose to develop a position-sensitive detector consisting of a monolithic scintillating crystal on which a high efficiency photocathode is deposited (Figure 1).

This "scintronic" crystal, which combines scintillation and photoelectron generation, optimizes the transmission of scintillation and Cherenkov photons to the photocathode without any optical coupling media (e.g. optical grease). Such a device will avoid internal reflection of optical photons on the crystal/photocathode interface thanks to the high refractive index of the photocathode.

The "scintronic" crystal will be encapsulated within a micro-channel plate based multiplier tube (MCP-MT) to amplify the signal and optimize the transit time of the photoelectrons towards the detection anodes, thus minimizing the time resolution of the detection chain.

The elegance of our detector consists in:

- improving the efficiency of light collection in a scintillating crystal of high density and high effective atomic number by depositing a photocathode directly on the surface of the scintillating crystal,

- encapsulating this "scintronic" crystal with a MCP-MT,

- making use of the Cherenkov light emission to improve time resolution of the detector,

- utilizing the map of photoelectrons produced at the photocathode to reconstruct the properties of the gamma ray interaction by means of robust statistical estimators and multivariate analysis.

We propose (Figure 1) to use a scintillating crystal with low light production yield $(\sim 300$ photons/MeV), but very fast (main decay time of a few nanoseconds). This will allow us to detect Cherenkov photons without being dazzled by the slower scintillation light.
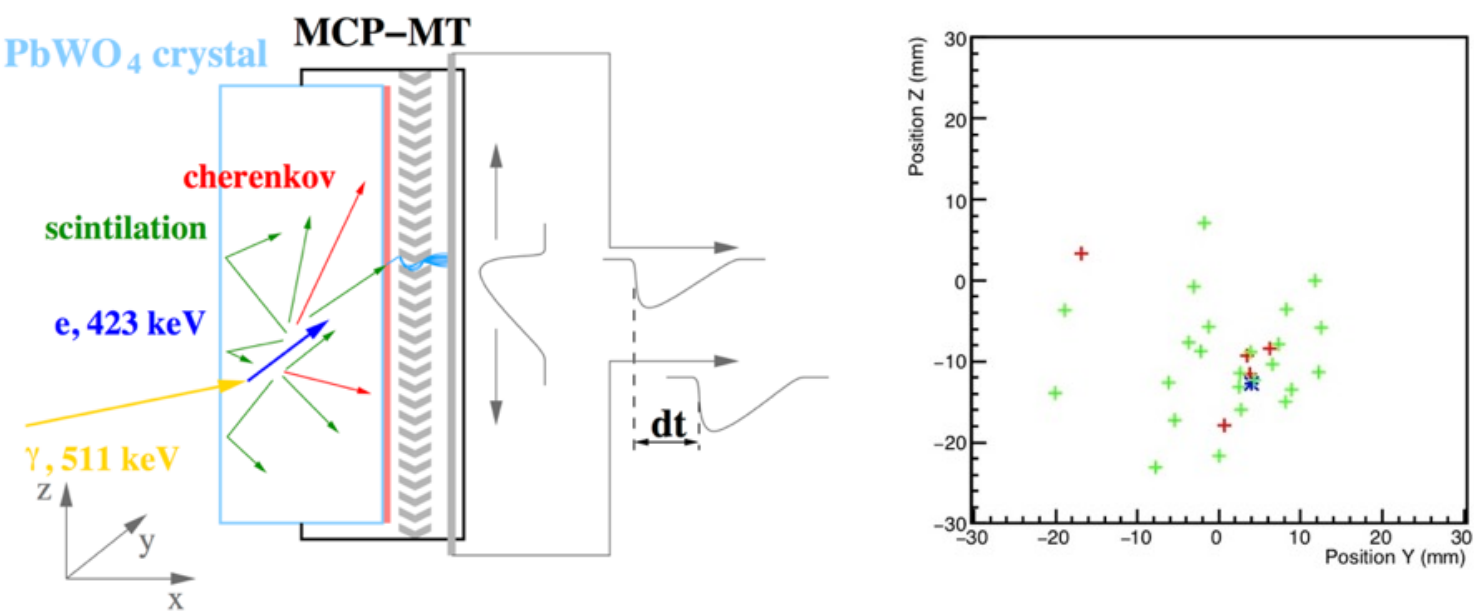

Figure 1: (Left) "Scintronic" crystal encapsulated within a MCP-MT. The photocathode is deposited directly on the scintillating crystal. The generated photoelectrons are amplified by a micro-channel plate. The amplified signals are collected on a densely pixelated anode plane read out by transmission lines and fast electronics [Kim, 2012]. (Right) Example of a photoelectron map produced on the photocathode by $511 \mathrm{keV}$ gamma ray interactions within the crystal. The blue crosses mark energy deposits in the crystal, the red ones 
correspond to the photons detected during the first 500 ps (mainly produced by Cherenkov effect) and the green ones mark scintillation photons detected between 500 ps and $20 \mathrm{~ns}$

For this, we have measured the vapor pressures of the decomposition products of several crystals, "Cherenkov radiators" or scintillators, heated under vacuum (i.e. the process used for the photocathode deposition). As a result, we have selected lead tungstate $\left(\mathrm{PbWO}_{4}\right)$, which should allow to deposit the photocathode (e.g. bialkali, multialkali, etc.) directly on the scintillating crystal. The crystal would thus become the entrance window of the photomultiplier.

Depositing the photocathode directly on the scintillating crystal allows one to avoid the use of an optical coupling technology (e.g. optical gel) with a refractive index close to 1.5 and that of $\mathrm{PbWO}_{4}$ is 2.3. This optical gel induces a strong loss of light by total reflection at the crystal/gel interface. The calculated total reflection angle value is $41^{\circ}$. This implies that assuming diffuse light is impinging at the optical interface, more than $75 \%$ of the photons lie in the solid angle where optical photons undergo total reflection. The photocathode refractive indices measured in the absorption band are close to 2.7. When the photocathode is deposited on the crystal, the transmission of photons from the crystal to the photocathode happens without total reflection. Hence, we expect a gain in the optical photon transmission probability by a factor up to 4 .

The gain in optical coupling allows optimizing the time measurement based on Cherenkov light. The presence of a small number of scintillation photons associated with the use of a MCP and a densely pixelated anode plane makes it possible to acquire an image of the time-position map of photoelectrons produced on each instrumented face of the detector bloc (see Figure 1 right and Figure 7). This detector design has been patented [37].

\section{i. Photocathode on scintillating crystal}

The first technological issue is the production of an efficient and stable photocathode on the surface of a $\mathrm{PbWO}_{4}$ crystal. The reference in terms of efficiency to visible wavelengths $(\geq 350 \mathrm{~nm})$ remains the bialkali and multi-alkali photocathodes. However, these photocathodes are instantly deteriorated in contact of oxygen, even at very low concentrations. Therefore, the realization and the assembly of the components of the detector must take place under high vacuum.

\section{ii. Producing large size crystals}

The second technological issue consists in producing large-sized $\mathrm{PbWO}_{4}$ scintillating crystals up to $60 \times 60 \times 20 \mathrm{~mm}^{3}$. We have identified 3 companies producing $\mathrm{PbWO}_{4}$ scintillation crystals: the Czech company CRYTUR (Turnov), and the Chinese companies SICCAS (Shangai) and EPIC (Jiangsu).

\section{iii. Signal readout}

Finally, the third technological issue is related to the number of pixels to read out on the anode plane of the MCP-MT: a matrix of $1024(32 \times 32)$ pixels or channels. To control the complexity, the heat dissipation and the electronics cost, we have connected these anodes to 32 transmission lines that are read out at both ends. The time difference in signal arrivals from both the edges allows to reconstruct the coordinate along the line and thus to divide the number of the channels by a factor 16. [38] have developed such a readout by using transmission lines to instrument high-yield scintillating crystals coupled with optical gel to micro-channel plate photomultiplier tubes (MCP-PMTs). They have shown that this configuration makes it possible to reconstruct the signals of the anode plane without measurable degradation of the MCP-PMT time resolution.

\section{iv. Photocathode deposition on $\mathrm{PbWO}_{4}$ crystal}

The deposition of a photocathode layer on a $\mathrm{PbWO}_{4}$ crystal was carried out by the Bristish Photek company (St Leonards on Sea). After several tests, the work resulted in a great success: Photek has achieved a photocathode deposition on $\mathrm{PbWO}_{4}$ with a quantum efficiency of $25 \%$ at $400 \mathrm{~nm}$. 

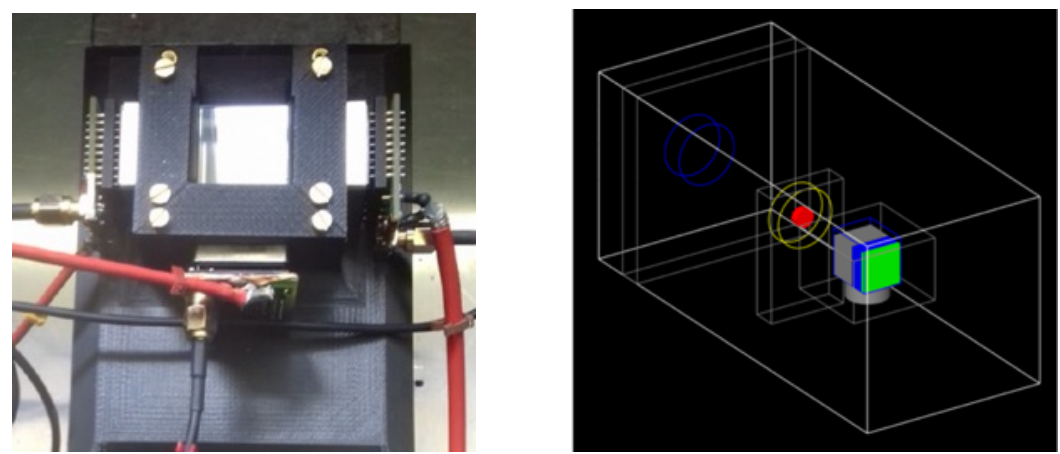

Figure 2: Crystal scintillation properties measurements. (Left) Picture of a cubic $\mathrm{PbWO}_{4}$ crystal coupled with 3 Hamamatsu R11256-100 photomultipliers using optical gel. (Right) Simplified Monte Carlo simulation layout of the light yield measurement setup. We have used a $25 \times 25 \times 10 \mathrm{~mm}^{2} \mathrm{PbWO}_{4}$ crystal grounded on all its faces, but the one coupled to the photomultiplier. We used a ${ }^{22} \mathrm{Na}$ radioactive so urce and an additional YAP detector to tag the $511 \mathrm{keV}$ photons from positron annihilations.

\section{v. $\mathrm{PbWO}_{4}$ crystals light yield}

We have measured and then compared scintillation light production yield and decay times of 3 $\mathrm{PbWO}_{4}$ crystals with different doping from different suppliers (Table 2). The setup is shown at Figure 2. All the 3 crystals show quite similar performances consistent with our expectations. We are currently refining these measurements and preparing a dedicated paper documenting a temperature dependence study similar to the one performed for the Panda2 collaboration [36] and time constant measurements.

Table 2: Scintillation yield expressed as the ratio of the number of photoelectrons measured at room temperature with $3 \mathrm{PbWO}_{4}$ crystals to that calculated by Monte Carlo, assuming a production of 300 photons/MeV with a $\mathrm{PbWO}_{4}$ : $\mathrm{Y}$ emission spectrum (work in progress).

\begin{tabular}{|c|c|}
\hline Technology & $\begin{array}{c}\text { Scintillation yield } \\
(\%)\end{array}$ \\
\hline Crytur: Panda2 & 0.97 \\
\hline SICCAS: CMS & 0.81 \\
\hline SICCAS: Y-doped & 0.87 \\
\hline
\end{tabular}

\section{vi. MCP-PMT and transmission lines readout}

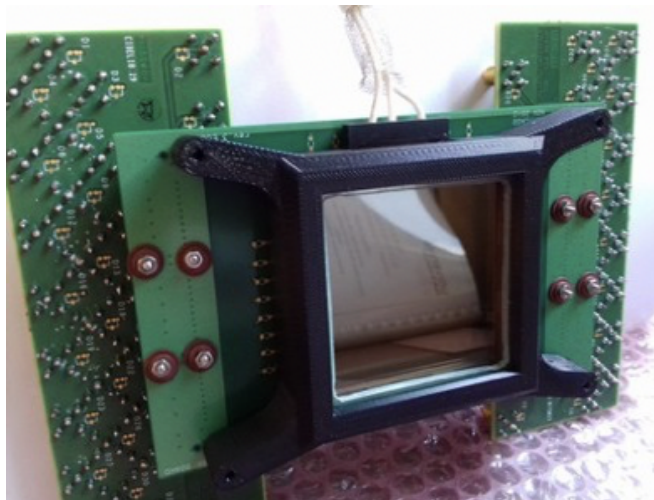

Figure 3: Picture of the MCP-PMT photodetector used for these developments.

A planacon XP-85122 MCP-PMT with $10 \mu \mathrm{m}$ pore size and $1024(32 \times 32)$ anode pads was mounted on a transmission line readout board (Figure 3), provided by the university of chicago. The printed circuit board (PCB) was attached to the multi-pixelated anode pads of $1.1 \times 1.1 \mathrm{~mm}^{2}$ using a roomtemperature bonded $3 \mathrm{M}^{\mathrm{TM}}$ conductive anisotropic tape (reference number 9705). Two additional boards were adapting the high density SAMTEC connector outputs to the $50 \mathrm{ohms} \mathrm{SMA} \mathrm{standard,} \mathrm{which} \mathrm{is}$ more convenient for small scale prototyping. 

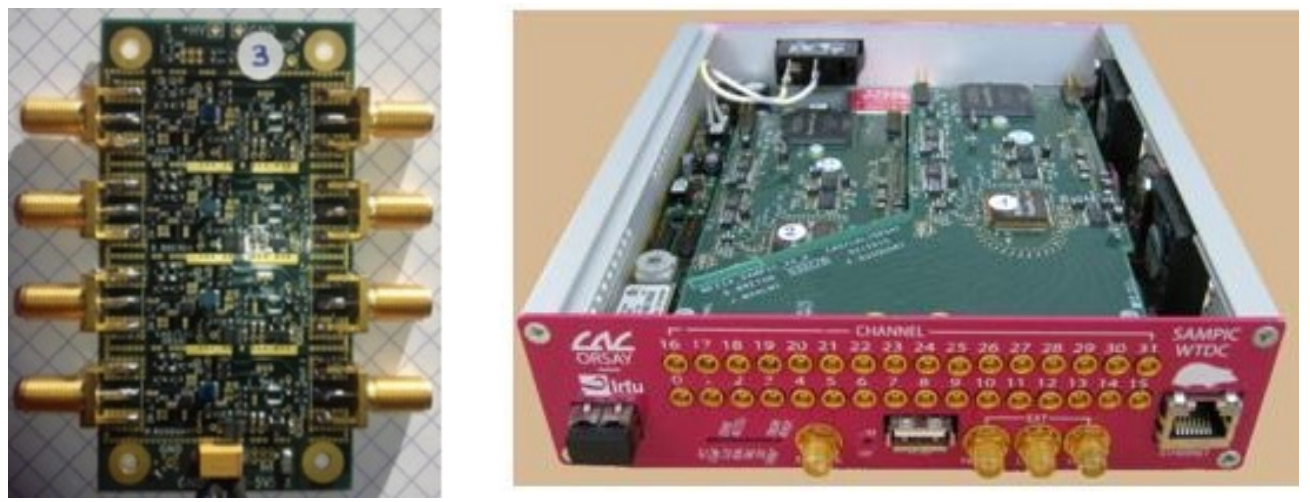

Figure 4: (Left) 4-channels fast amplifier prototype with a $700 \mathrm{MHz}$ bandwidth and a gain of 100 used for the tests described in this work. (Right) 32-channels SAMPIC waveform recorder, which is currently used on many test benches all around the world [SAMPIC Workshop].

\section{vii. Fast readout amplifiers}

We have developed prototypes of a fast multichannel amplifier board based on a broadband RFIC amplifier. The latter permits amplifying very fast negative pulses with a gain of $20 \mathrm{~dB}$ on $50 \mathrm{ohms}$ with a moderate power supply (18 mA per channel). The measured bandwidth of $700 \mathrm{MHz}$ offers a good compromise between noise and rise time for this detector. Special attention was paid to the channel powering and the PCB design in order to ensure a very low crosstalk between the channels. The 4channel amplifier board shown in Figure 4 (left) houses two amplifying stages, hence presenting a gain of $40 \mathrm{~dB}$.

\section{viii. SAMPIC waveform recorder}

The 32-channels SAMPIC module (Figure 4, right) used in this setup is based on two 16-channels SAMPIC_V3C chips, themselves based on the new patented concept of waveform and time-to-digital converter (WTDC) [40], [41]. Each channel of the chip includes a DLL-based TDC to provide a rough time associated with an ultra-fast analog memory, which samples the signals between 1.6 and $8.5 \mathrm{GS} / \mathrm{s}$. The recorded waveforms are used for precise timing measurements with a rms resolution of a few picoseconds. Every channel integrates a discriminator that can trigger it independently or participate to a more complex trigger. A first trigger level is implemented on-chip, while a second trigger level can be performed at the module level (32 channels). An associated data acquisition software is used to configure the module, start and stop acquisitions, store recorded data on disk in binary or ASCII format and visualize signal waveforms and parameter distributions. The SAMPIC module transfers raw signal waveforms to the computer, where all the necessary calibrations are applied on the fly by software. In addition, the latter performs one of the three hit time extraction algorithms: fixed threshold, constant fraction discriminator (CFD) or multiple CFDs [42]. In this work, we have run at $6.4 \mathrm{GS} / \mathrm{s}$ and used the CFD algorithm with a ratio of 0.5. Data stored on disk include signal waveforms (optional), calculated times and amplitudes, and selected SAMPIC parameters.

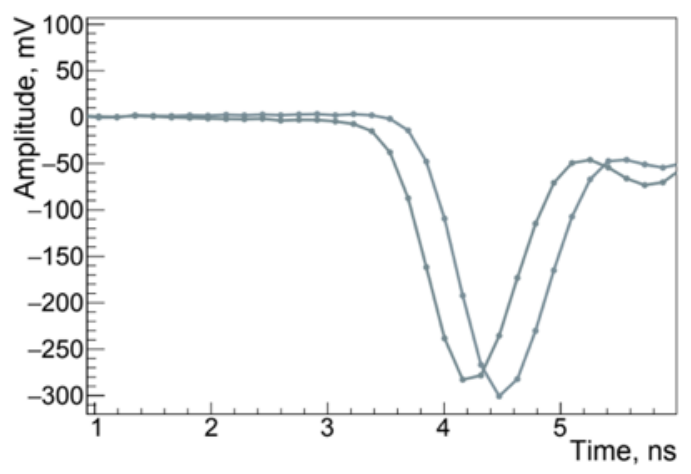


Figure 5: Single photoelectron as detected by the XP-85122 MCP-PMT. The pulses are registered by the SAMPIC Waveform recorder at both ends of a readout transmission line. Before the pulse, the signal shape is very flat, showing that the readout noise is minimal.

\section{ix. Photodetector performance}

For a first test of this readout paradigm, we have acquired data from eight transmission lines. All the other transmission lines were grounded through $50 \mathrm{ohms}$ at both ends. The MCP-PMT was mounted on a X-LRT-C computer-driven micrometric translation table from Zaber Technologies (Vancouver, Canada). A picosecond laser of type Pilas manufactured by the company NKT Photonics (Cologne, Germany) configured to generate single photoelectrons was lightening the MCP-PMT through a $300 \mu \mathrm{m}$ pinhole placed at $2 \mathrm{~mm}$ of the optical window. This system allowed us to precisely position and move the MCP-PMT behind the pinhole.

We have measured very accurately the time differences between the two signals at the ends of the readout line for a pinhole centered on a readout line (Figure 5). The time difference distribution is gaussian and the measured widths range from 16 to 20 ps FWHM, depending on the readout line measured, but close to the SAMPIC module time resolution limit. From the measured signal speed along the readout line, we have determined spatial resolutions along the line that amount to 1.2 to $1.5 \mathrm{~mm}$ FWHM (Figure 6, left).
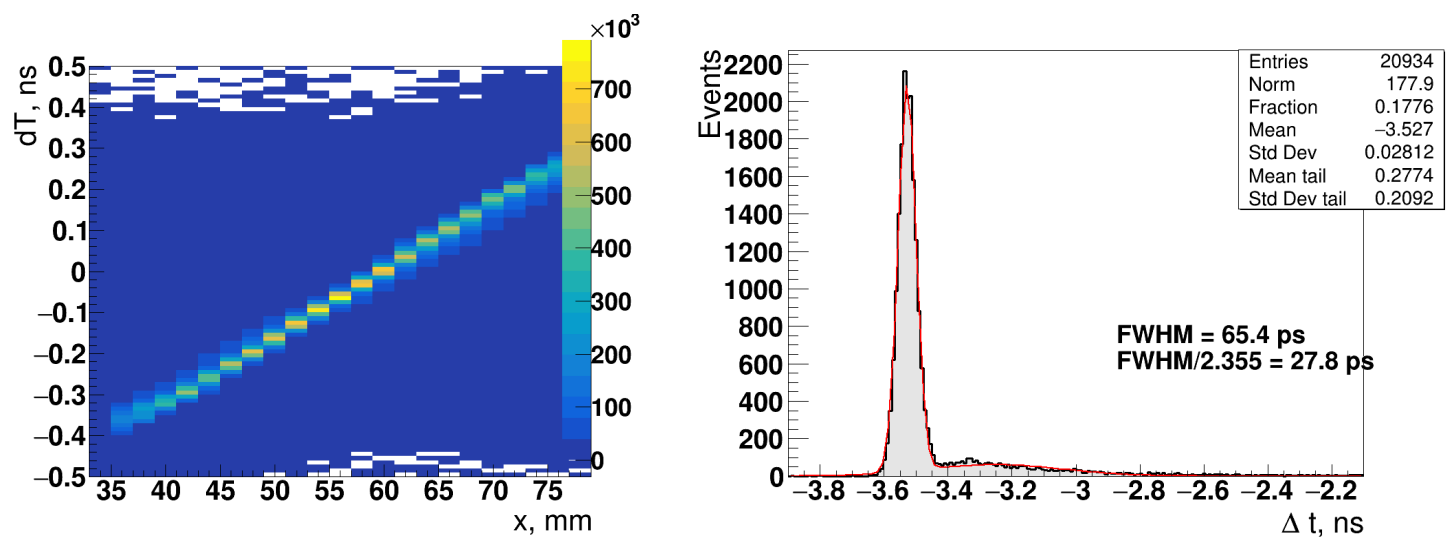

Figure 6: (Left) Histogram of position of the pinhole along the line versus time difference between signals registered at both ends of the targeted transmission line. (Right) Histogram of the time difference between the

laser pulse and the average of the pulse times measured at both ends of the transmission lines.

The average of the pulse times measured at both ends of the transmission lines provides an accurate measurement of photoelectron detection time. The histogram of the time difference between the readout lines and the laser pulse, (Figure 6, right) shows a narrow main peak with a width of 67 ps FWHM and a long tail, typical of a photoelectron back-scattered at the entrance of MCP-PMT, which corresponds to $25 \%$ of the total statistics.

\section{Simplified detector Monte Carlo simulation}

In order to optimize detector design and prepare future data analysis, we have written a simplified detector Monte Carlo simulation, which will be updated and refined as the hardware will evolve. We have used the CERN GEANT4 library [43] interfaced through the GATE macro language and libraries [29], [30]. We have assumed that the scintillating crystal will consist of a monolithic $\mathrm{PbWO}_{4}$ crystal $60 \times 60 \times 10$ (or 20) $\mathrm{mm}^{3}$. Crystal properties were extracted from the literature, choosing $\mathrm{PbWO}_{4}: \mathrm{Y}$ as a reference for this study. The refraction index was simulated according to [44]. We have averaged the ordinary and extraordinary refraction index in order to code mean optical propagation properties. The scintillation wavelength distribution was extracted from [45]. The attenuation length in $\mathrm{PbWO}_{4}$ as function of the scintillation wavelength was modelled according to [35]. The $\mathrm{PbWO}_{4}$ light yield was assumed to be 300 photons/MeV from data published in [46] and [33]. 
The two $60 \times 60 \mathrm{~mm}^{2}$ opposites faces of the crystal are covered by a blue-bialkali photocathode directly deposited on the crystal surface (first detector prototype will have only one). All the other crystal faces are assumed to be polished and black. The photocathode model, namely its refraction index, absorption length, and photoelectron production and extraction efficiencies were taken from [Motta, 2005].

$511 \mathrm{keV}$ gamma rays impinge on the crystal surface ( $\mathrm{Y}$ and $\mathrm{Z}$ axis) and propagate along the $\mathrm{X}$ axis. Simulation of gamma ray interactions uses the Livermore library. Optical photons were produced by both the Cherenkov effect and the $\mathrm{PbWO}_{4}$ scintillation.

GATE was configured to save data in the GEANT4/ROOT HitsFile format. A dedicated C++ software was then written to gather output data and store the time, 3D coordinates and energy of every gamma ray interaction within the crystal. Then, the absorption of optical photons (position, time and deposited energy) in the crystal and at the photocathodes were registered. This software also models the behavior of the photocathode according to [47] and computes the foreseen photoelectron production (time and 2D coordinates). This preliminary simulation did not include a model neither for the MCPPMT photodetector, nor for the readout electronics.
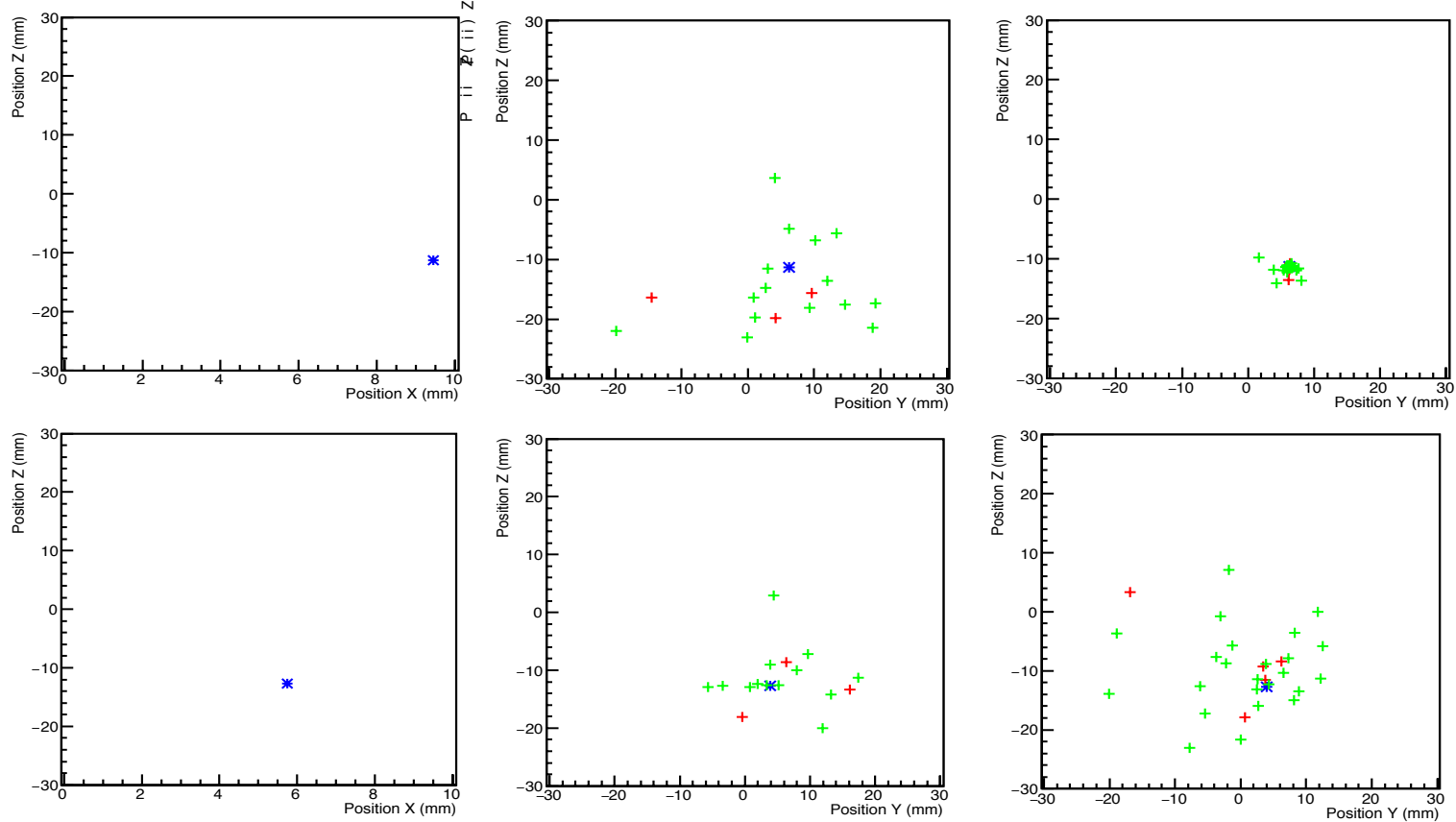

Figure 7: Simulated data for a $10 \mathrm{~mm}$ thick "scintronic" crystal instrumented on two sides by a bialkali photocathode. $511 \mathrm{keV}$ gamma rays impinge on the detector from the left and propagate along the $\mathrm{X}$ axis. The upper and lower rows correspond to the photoelectric interaction of gamma rays close to the surface of the crystal and at mid-crystal depth, respectively. Notice the coordinates axis of each of the figures.

(left) Blue crosses denote gamma ray interactions within the crystal. (centre and right) show photoelectrons produced on the two photocathodes. Red and green crosses mark photoelectrons produced at $\mathrm{X}=0 \mathrm{~mm}$ (center) and $\mathrm{X}=10 \mathrm{~mm}$ (right) during the first $500 \mathrm{ps}$ and the following $20 \mathrm{~ns}$, respectively.

Figure 7 shows the computed photoelectron maps produced at the photocathode layers, which will be referred to as hit-maps. Typically, for a photoelectric interaction of a $511 \mathrm{keV}$ gamma ray within $\mathrm{PbWO}_{4}$, according to Geant4, under the assumptions stated above $\left(\mathrm{PbWO}_{4}: \mathrm{Y}\right.$ optical transparency stopping at $350 \mathrm{~nm}$, refraction index varying from 2.3 to 2.1 for optical wavelength), 20 Cherenkov photons and 150 scintillation photons are produced. Monte Carlo simulation predicts an average of 20 photoelectrons produced by each photocathode. When an interaction takes place close to the surface of the detector, the hit-map corresponding to this face is very dense. In contrast, the hit-map corresponding to the opposite face of the crystal is spread on a large area. When the interaction takes place at midcrystal depth, the apparent density of both hit-maps look intermediate. 
Quantifying the properties of these hit-maps (barycenter, dispersion of hits around the barycenter, photon detection times, etc.) can then be used to assess the spatial and temporal properties of gamma ray interactions within the crystal (hidden physical information of interest).

\section{i. Preliminary event reconstruction}

In this preliminary study, we have quantified on the hit-maps the number of photoelectrons, the 2D barycenter of the photoelectron positions, the 2D spread of the photoelectron positions around the barycenter, the time of the first photoelectron and the time distribution of prompt (within the first $500 \mathrm{ps}$ ) photoelectrons.

From the gamma ray interaction data, we have extracted the 3D coordinates and the time of the first gamma ray interaction, the total energy deposited in the crystal, and when there was more than one interaction, the barycenter of interaction positions weighted by the energy deposited at each interaction point.

Because of the low scintillation yield of $\mathrm{PbWO}_{4}$, the number of photoelectrons provide a measurement of the energy deposited in the crystal of modest accuracy ( $\sim 25 \%$ FWHM), but compatible with a use in a PET scanner.

Classical (i.e. moment based, gaussian statistics) estimators (e.g. mean, variance) applied to hitmaps turned not to correlate accurately with the $3 \mathrm{D}$ coordinates of the gamma ray interactions. This is because, as it can be guessed from Figure 7, photoelectron distributions display long tails that impact strongly the computation of statistical moments. More "robust" statistical estimators have then to be used, the simplest being the medians of the distributions. Indeed, a barycenter defined as the median of the photoelectron coordinates along the $\mathrm{Y}$ and $\mathrm{Z}$ axis shows significantly improved correlations with the gamma interaction position. We have used:

$$
Y_{\text {bary }}=\operatorname{median}\left\{Y_{P E i}\right\}, Z_{\text {bary }}=\operatorname{median}\left\{Z_{P E i}\right\}
$$

This is also true when quantifying the spread of hit-maps, for which we have used:

$$
Y_{S p r}=\operatorname{median}\left\{\left|Y_{P E i}-Y_{b a r y}\right|\right\}, Z_{S p r}=\operatorname{median}\left\{\left|Z_{P E i}-Z_{b a r y}\right|\right\}
$$

From these values, we define the radial spread as:

$$
\operatorname{RadSpr}=\left(Y_{S p r}{ }^{2}+Z_{S p r}{ }^{2}\right)^{1 / 2}
$$
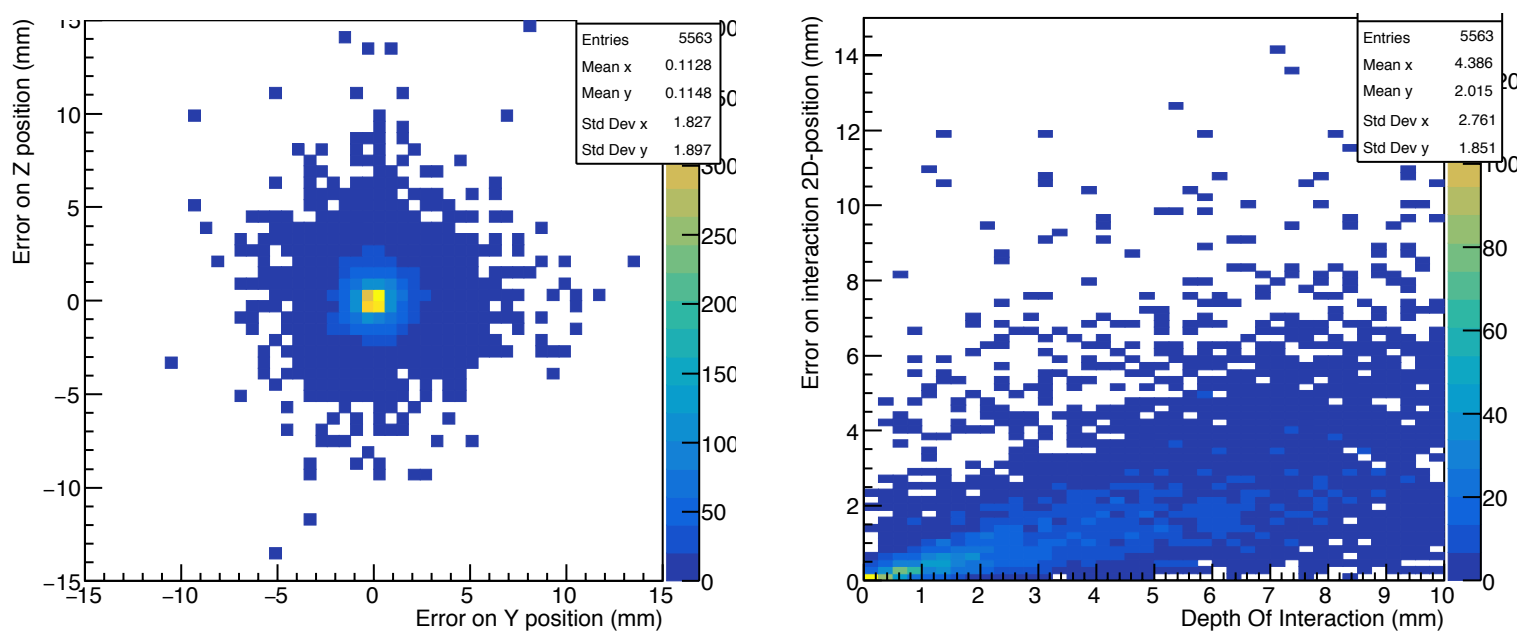

Figure 8: (Left) 2D histogram of the difference of computed $Y$ and $Z$ coordinates of the PE barycenter and the gamma ray position, using robust statistics. (Right) 2D histogram of DistbaryPE (see text for the definition) versus DOI. In these figures, we have used data from the front photocathode only. No selections were applied on the energy deposited in the $\mathrm{PbWO}_{4}$ crystal. 


\section{ii. Computed performance}

In order to study the foreseen accuracy of a full (two faces instrumented) detector, we have assumed a $60 \times 60 \times 10 \mathrm{~mm}^{3} \mathrm{PbWO}_{4}$ crystal. We have simulated $511 \mathrm{keV}$ gamma rays impinging orthogonally on the front face of the crystal at positions uniformly distributed within a centered square of $30 \times 30 \mathrm{~mm}^{2}$. This allows a preliminary study of the detector performances, without introducing the additional complexity of border effects. We have then computed the 2D (Y-Z coordinates) distance between the photoelectron barycenter and the gamma ray interaction position Dist baryPE$_{\text {. }}$.

$$
\text { Dist }_{\text {bary } P E}=\left(\left(\mathrm{Y}_{\text {bary }}-\mathrm{Y}_{\text {gamma }}\right)^{2}+\left(\mathrm{Z}_{\text {bary }}-\mathrm{Z}_{\text {gamma }}\right)^{2}\right)^{1 / 2}
$$

Figure 8 quantifies the correlation of Dist $\mathrm{bary}_{\mathrm{PE}}$ to the gamma ray interaction position, using data from the front photocathode $(X=0)$ only. As it could be guessed from Figure 7, the larger the distance of the gamma ray interaction from the photocathode layer, the less accurate the reconstruction of the position.

The same study was made on the correlation between the measured radial spread, the time difference between the first photoelectrons detected by the photoelectric layers and the DOI of the gamma interaction within the crystal. Figure 9 shows some results.
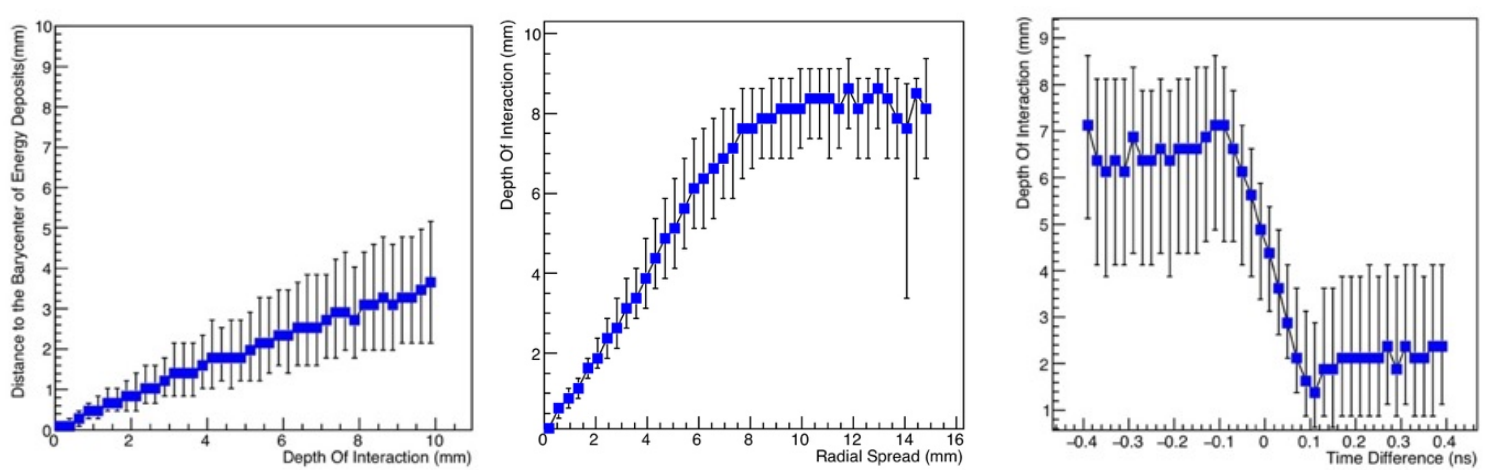

Figure 9: (Left) DistBaryPE versus DOI. (Center) DOI as function of hit-map radial spread. (Right) DOI as function of time difference between the first photoelectron detected on the front and the rear photocathodes. For these three graphs, points show the mean values, bars on points correspond to the quartile value of the distribution statistics. No selections on energy deposited in the crystal were made.

From Figure 9, we notice that the gamma ray interaction parameters correlate with the detector observables in a complex way and that none of these observables are accurate enough to provide alone an accurate estimation of gamma ray interaction parameters. Left figure shows that the 2D (Y-Z coordinates) gamma ray interaction positioning accuracy depends a lot on the distance of the interaction to the photocathode. Center and left plots show the complex correlation between the gamma ray DOI (parameter to be reconstructed) and some of the detector observables.

Nevertheless, there are many observables into which the information is encoded. In order to extract more efficiently those physical parameters, we have chosen to use the resources of machine learning algorithms.

For a quick overview of available machine learning methods available, we have used the Toolkit for Multivariate Data Analysis (TMVA V4.2.0) library [48] as delivered within the ROOT modular scientific software toolkit package [49]. This package turned out to be very convenient: once the data have been preprocessed and formatted as a spreadsheet, we were able to select a collection of algorithms and to get a quick assessment of their efficiencies within few days. In this preliminary study, we have selected the KNN (k-Nearest Neighbour), FDA-GA (Functionnal Discriminant Analysis), MLP (MultiLayered Perceptron) and Boosted Decision Tree (BDTG) methods. We have focused this work on the reconstruction of the 3D-coordinates, time and energy of the gamma ray interactions within the crystal. 
We have assumed that a photocathode was deposited and instrumented directly on the front and rear faces of the crystal. Then we have fed the algorithms with the following statistical variables extracted from the simulated hit-maps:

- $\quad Y_{\text {bary }}, Z_{\text {bary }}, Y_{S p r}, Z_{\text {spr }}$

- the time of the first detected photoelectron

- the number of fast photoelectrons (i.e. detected within the first $500 \mathrm{ps)}$

- the number of slow photoelectrons (i.e. detected after $500 \mathrm{ps)}$

to which we have added the total number of photoelectrons detected in the event.

We have found that MLP and BDTG performed best with our simulated data giving very similar performances. MLP required a significant computation time, whereas BDTG procured a significantly faster training time, but its training algorithm happened to break with the library version we have used. It has shown also a tendency to do overtraining. Therefore, for the results which will follow, we have chosen to use a MLP based algorithm, i.e. a classical neural network structure.

In order to minimize the complexity of the work, we have decided to train a dedicated network for each parameter of the gamma ray interaction we were aiming at. This allows to simplify the network structure while keeping good performances. We have tried a handful of configurations, selecting the one that has provided fast training times and good performances. Hence, we have chosen a structure with two hidden layers and the hyperbolic tangent activation function, resulting in a $15 \times 5 \times 3 \times 1$ network structure. In this configuration on a recent laptop computer (thus without using GPU), we needed $22 \mathrm{~s}$ CPU time for the MLP algorithm training and $7.2 \mu \mathrm{s} /$ event for event processing. Computer processing time for event reconstruction is thus not foreseen to be an issue with such an algorithm.
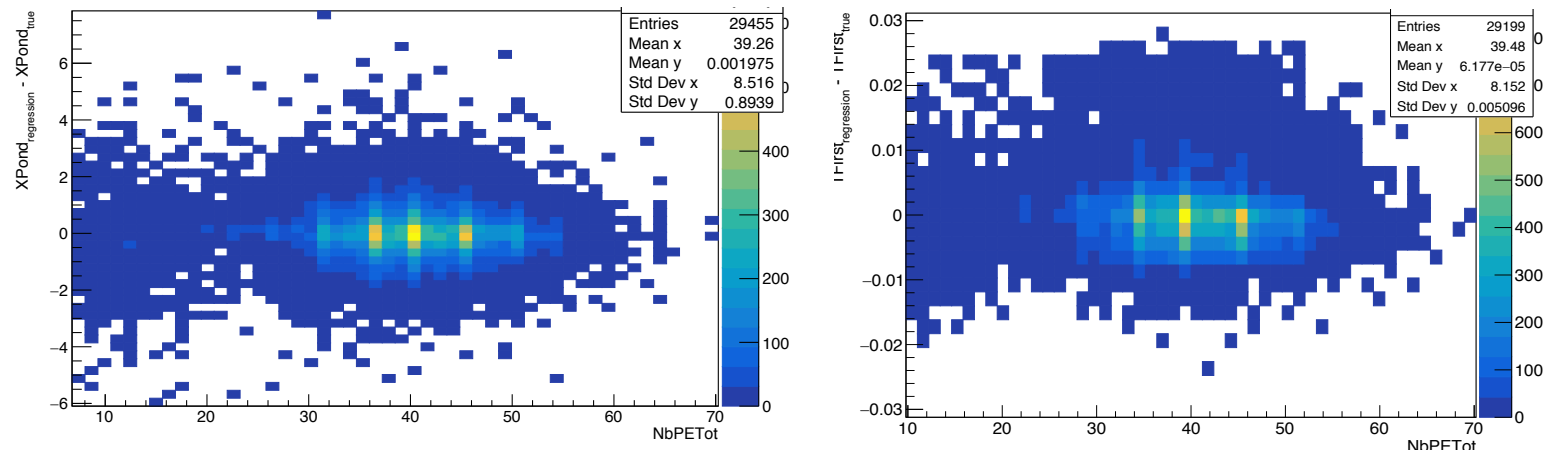

Figure 10: Evaluation of the performances of the MLP algorithms to reconstruct DOI and gamma ray interaction time within the $\mathrm{PbWO}_{4}$ crystal from hit-map data. (Left) 2D histogram of the number of detected photoelectron versus the difference between reconstructed and simulated DOI (mm). (Right) 2D histogram of the number of detected photoelectron versus the difference between reconstructed and simulated time of the first gamma ray interaction (ns) within the crystal. No selection cuts were applied on simulated data.

Figure 10 shows 2D histograms of the reconstructed gamma ray interaction parameters versus the total number of photoelectrons produced in the photocathode. Both the histograms show very accurate performances, though simulated events include Compton and multiple interactions within the crystal.

Table 3: Average deviation (rms) computed on the DOI, lateral position, and time of the first gamma ray interaction with a MLP algorithm fed with robust statistics estimators. The first line gives the standard deviations computed from the complete statistics. The second line computes these standard deviations after removing the distribution tails larger than $2 \sigma$.

\begin{tabular}{|c|c|c|c|c|}
\hline $\begin{array}{c}\text { Reconstructed parameter } \\
(\mathrm{rms})\end{array}$ & $\begin{array}{c}\text { DOI } \\
(\mathrm{mm})\end{array}$ & $\begin{array}{c}\text { Y coordinate } \\
(\mathrm{mm})\end{array}$ & $\begin{array}{c}\text { Time of the first gamma } \\
\text { ray interaction }(\mathrm{ps})\end{array}$ & $\begin{array}{c}\text { Energy deposit } \\
(\mathrm{keV})\end{array}$ \\
\hline Complete statistics & 0.91 & 1.57 & 5.5 & 27 \\
\hline Truncated statistics & 0.69 & 1.28 & 4.2 & 7.3 \\
\hline
\end{tabular}

Table 3 quantifies the results for all the gamma ray interaction parameters. The predicted accuracies are very motivating, though the simulated events include Compton and multiple interactions 
within the crystal. However, the energy deposit accuracy computation should be taken carefully. Indeed, the neural network has been trained assuming that all the impinging gamma rays had an energy of $511 \mathrm{keV}$. Thus, above the Compton edge, most energy deposits amount to $511 \mathrm{keV}$ in the training sample and are identified as such, with zero reconstruction error in the test sample. Thus the computed energy resolution in this simulation is not representative of the energy resolution of the detector if it would have to be tested with random energy radioactive sources.

The reconstruction of the first gamma ray interaction time is very accurate and amounts to $13 \mathrm{ps}$ FWHM, showing that the MLP algorithm is able to decorrelate appropriately time information from DOI. Nevertheless, our simulation was implementing neither the photodetector time transit spread, nor the readout electronics noise. Indeed, good quality MCP-PMT have single photoelectron time transit spread of 70 ps FWHM with extra $25 \%$ events in a tail at the nanosecond time scale. Therefore, the above computed time resolution is quite optimistic and will be studied in details in future work. Forecasting its value will require detailed modelling of the full experimental readout chain. Nonetheless, this preliminary study has shown that machine learning algorithms have the potential to reconstruct accurately gamma ray interaction parameters when they are trained upon accurate Monte Carlo simulation.

\section{Discussion}

In this paper, we propose a new concept of a "scintronic" PET detection module. We show that the main key technologies for its assembly have been demonstrated and we present a Monte Carlo study showing the strong potential of this "scintronic" detector design in terms of time resolution, efficiency and spatial resolution.

Further work will consist in assembling a detector with a relatively thin $(\sim 5 \mathrm{~mm}) \mathrm{PbWO}_{4}$ scintillating crystal used as the input window of a standard 2" squared MCP-MT. This design will allow us to validate the full "scintronic" detector assembling process, to learn how to optimize signal readout both from the hardware and software points, and hopefully to achieve competitive detector performance in terms of time and spatial resolutions. For detectors thicker than $5 \mathrm{~mm}$, time resolution and overall event reconstruction performance will significantly improve when both the front and the rear faces of the $\mathrm{PbWO}_{4}$ crystal will be instrumented. However, we foresee that integrating a thick scintillating crystal in between two MCP-PMTs will be difficult and therefore, we are also considering to use either a SiPM matrix or a regular MCP-PMT assembled with a standard optical coupling for the second face.

\section{Acknowledgments}

The authors would like to thank J.-Ph. Renault from the CEA Saclay-IRAMIS and S. Chatain from CEA Saclay-DES for many advices related to chemistry and for their measurements, which have driven our choice of the detector crystal. We want to thank Prof. H. Frisch from Chicago University for stimulating discussions and for providing prototypes of transmission line readout boards. We also wish to thank Prof. M. Korjik from the Belarusian State University for discussions and advices on $\mathrm{PbWO}_{4}$ scintillating crystal growth technologies. Finally, we wish to thanks R. Trebossen, S. Jan and C. Comtat from CEA-SHFJ for guiding us in the world of medical physics, and the Referee for his detailed review of the article.

We are grateful for the support and seed funding from the CEA "Programme Exploratoire BottomUp" under grant No. 17P103-CLEAR-MIND and the French National Research Agency under grant No. ANR-19-CE19-0009-01.

\section{References}

[1] A. Iltis and H. Snoussi, "The Temporal PET Camera: A New Concept With High Spatial and Timing Resolution for PET Imaging,” J. Imaging, 1 (2015), 45.

[2] D.R. Schaart et al., "LaBr3:Ce and SiPMs for time-of-flight PET: achieving 100 ps coincidence resolving time," Phys. Med. Biol., 55 (2010) N179. 
[3] G. Borghi et al., "A $32 \mathrm{~mm} \times 32 \mathrm{~mm} \times 22 \mathrm{~mm}$ monolithic LYSO:Ce detector with dual-sided digital photon counter readout for ultrahigh-performance TOF-PET and TOF-PET/MRI," Phys. Med. Biol., 61 (2016) 4929.

[4] S. Liprandi et al., "Sub-3mm spatial resolution from a large monolithic $\mathrm{LaBr}_{3}(\mathrm{Ce})$ scintillator," $C D B M E, 3$ (2017) 655.

[5] M. Pizzichemi et al., "On light sharing TOF-PET modules with depth of interaction and 157 ps FWHM coincidence time resolution," Phys. Med. Biol., 64 (2019) 155008

[6] S. Gundacker et al., "Experimental time resolution limits of modern SiPMs and TOF-PET detectors exploring different scintillators and Cherenkov emission," Phys. Med. Biol., 65 (2020) 025001.

[7] R. Lecomte, “Novel detector technology for clinical PET,” Eur. J. Nucl. Med. Mol. Imaging, 36 (2009) S69.

[8] M. Conti and B. Bendriem, "The new opportunities for high time resolution clinical TOF PET," Clin. Transl. Imaging, 7 (2019) 139-147.

[9] S. Surti et al., "Performance of Philips Gemini TF PET/CT scanner with special consideration for its timeof-flight imaging capabilities," J. Nucl. Med., 48:3 (2007) 471-480.

[10] B.W. Jakoby et al., "Physics performance and clinical investigation of the mCT time-of-flight PET/CT scanner," Phys. Med. Biol., 56 (2011) 2375-2389.

[11] D.F. Hsu et al., "Studies of a next-generation silicon-photomultiplier-based time-of-flight PET/CT system," J. Nucl. Med., 58 (2017) 1511-1518.

[12] I. Rausch et al., "Performance evaluation of the Philips Vereos PET/CT system according to the NEMA NU2-2012 standard," J. Nucl. Med., 60:4 (2019) 561-567.

[13] J.J. van Sluis et al., "Performance characteristics of the Digital Biograph Vision PET/CT system," J. Nucl. Med., 60:7 (2019)1031-1036.

[14] S.R. Cherry et al., "Total-body imaging: transforming the role of positron emission tomography," Sci. Transl. Med., 9 (2017) eeaf6169.

[15] D. Yvon et al., "Trimethyl Bismuth Optical Properties for Particle Detection and the CaLIPSO Detector," IEEE Trans. Nucl. Sci., 61 (2014) 60, 10.1109/TNS.2013.2291971.

[16] C. Grignon et al., "Nuclear medical imaging using $\beta+\gamma$ coincidences from ${ }^{44} \mathrm{Sc}$ radio-nuclide with liquid xenon as detection medium," Nucl. Instum. Meth. A, 571 (2007) 142.

[17] D.L. Snyder et al., "A mathematical-model for positron-emission tomography systems having time-of-flight measurements," IEEE Trans. Nucl. Sci,. 28 (1981) 3575-3583.

[18] T.F. Budinger, "Time-of-flight positron emission tomography - status relative to conventional PET," J. Nucl. Med., 24:1 (1983) 73-76.

[19] S. Vandenberghe et al., "Recent developments in time-of-flight PET," EJNMMI Physics, (2016) 3:3.

[20] P. Lecoq, "Pushing the limits in time-of-flight PET imaging," IEEE Tran.s Radiat. Plasma. Med. Sci., 1 (2017) 476-485.

[21] S. I. Kwon et al., "Bismuth germanate coupled to near ultraviolet silicon photomultipliers for time-of-flight PET," Phys. Med. Biol. 61 (2016) L38.

[22] S. E. Brunner and D. R. Schaart, "BGO as a hybrid scintillator / Cherenkov radiator for cost-effective timeof-flight PET," Phys. Med. Biol., 62 (2017) 4421. 
[23] E. Ramos et al., "Efficient and fast 511-keV $\gamma$ detection through Cherenkov radiation: the CaLIPSO optical detector," J. Instrum., 11 (2016) P11008, 10.1088/1748-0221/11/11/P11008.

[24] I. Somlai-Schweiger et al., "CHERENCUBE: Concept definition and implementation challenges of a Cherenkov-based detector block for PET," Med. Phys., 42 (2015) 1825.

[25] R. Ota et al., "Coincidence time resolution of 30 ps FWHM using a pair of Cherenkov-radiator-integrated MCP-PMTs," Phys. Med. Biol. 64 (2019) 07LT01

[26] S. Korpar et al., "Study of a Cherenkov TOF-PET module,” Nucl. Instum. Meth. A, 732 (2013) 595.

[27] C. Canot et al., "Development of the fast and efficient gamma detector using Cherenkov light for TOF-PET," J. Instrum., 12 (2017), C12029, 10.1088/1748-0221/12/12/C12029.

[28] C. Canot et al. "Fast and efficient detection of $511 \mathrm{keV}$ photons using Cherenkov light in PbF2 crystal, coupled to a MCP-PMT and SAMPIC digitization module," J. Instrum., 14 (2019), P12001.

[29] D. Strul et al., "GATE (Geant4 Application for Tomographic Emission): a PET/SPECT general-purpose simulation platform,” Nucl. Phys. B (Proc. Suppl.), 125 (2003) 75-79.

[30] S. Jan et al., "GATE V6: a major enhancement of the GATE simulation platform enabling modelling of CT and radiotherapy," Phys. Med. Biol., 56 (2011) 881, DOI: 10.1088/0031-9155/56/4/001.

[31] S. Jan et al., "GATE: a simulation toolkit for PET and SPECT,” Phys. Med. Biol., 49 (2004) 4543-4561.

[32] M. Alokhina, "Design of the Cherenkov TOF whole-body PET scanner using GATE simulation," PhD thesis, Univ. Paris-Saclay 2018: http://theses.fr/2018SACLS279.

[33] M. Nikl et al., "Influence of doping on the emission and scintillation characteristics of $\mathrm{PbWO}_{4}$ single crystals," J. Appl. Phys., 87 (2000) 4243.

[34] M. Shao et al., "Scintillation Characteristics of Doped PbWO4 Crystals," Chin. Phys. Lett., 18 (2001) 513.

[35] A.A. Annenkov et al., "Lead tungstate scintillation material," Nucl. Instum. Meth. A, 490 (2002) 30-50.

[36] A. Borisevich et al., "New Start of Lead Tungstate Crystal Production for High-Energy Physics Experiments," IEEE Trans. Nucl. Sci., 63 (2016) 569.

[37] D. Yvon and V. Sharyy, Brevet déposé, Réf : FR N 17/59065, 29 Sep. 2017.

[38] H. Kim et al., "A prototype TOF PET detector module using a micro-channel plate photomultiplier tube with waveform sampling," Nucl. Instum. Meth. A, 662 (2012) 26.

[39] WaveCatcher and SAMPIC International Workshop, LAL Orsay France, 7-8 Fev. 2018, https://wpsist.lal.in2p3.fr/wasiw2018/.

[40] D. Breton et al., Patent, Ref : WO2010149920A2; FR2947121A1; US2012187996A1; EP2446534A2.

[41] E. Delagnes et al., "Reaching a few picosecond timing precision with the 16-channel digitizer and timestamper SAMPIC ASIC,” Nucl. Instum. Meth. A, 787 (2015) 24.

[42] D. Breton et al., "Fast electronics for particle Time-Of-Flight measurement, with focus on the SAMPIC ASIC", Nuovo Cimento C, 43 (2020) 7. DOI: 10.1393/ncc/i2020-20007-6

[43] S. Agostinelli et al., “Geant4-a simulation toolkit,” Nucl. Instum. Meth. A, 506 (2003) 250-303.

[44] Y. Huang, "Luminescence Properties and Refractive-Index Characterization of Li+-Doped PbWO4 Single Crystals," J. Korean Phys. Soc., 50 (2007) 493.

[45] M. Shao et al., "Scintillation Characteristics of Doped $\mathrm{PbWO}_{4}$ Crystals," Chin. Phys. Lett., 18 (2001) 513.

[46] M. Kobayashi et al., "Significant improvement of $\mathrm{PbWO}_{4}$ scintillating crystals by doping with trivalent ions," Nucl. Instum. Meth. A, 434 (1999) 412. 
[47] D. Motta and S. Schönert, "Optical properties of bialkaly photocathodes," Nucl. Instum. Meth. A, $\mathbf{5 3 9}$ (2005) 217-234.

[48] A. Hoecker et al., "TMVA-Toolkit for Multivariate Data Analysis," PoS ACAT, (2007) 40, https://root.cern/tmva/.

[49] R. Brun and F. Rademakers, "ROOT - An Object Oriented Data Analysis Framework," Nucl. Instum. Meth. A, 389 (1997) 81-86, https://root.cern/. 